\title{
The tubular damage markers: neutrophil gelatinase- associated lipocalin and kidney injury molecule-1 in newborns with exposure to maternal diabetes during pregnancy
}

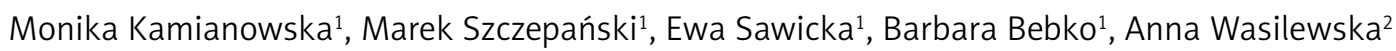

\author{
${ }^{1}$ Department of Neonatology and Neonatal Intensive Care, Medical University \\ of Bialystok, Bialystok, Poland \\ 2Department of Paediatrics and Nephrology, Medical University of Bialystok, Bialystok, \\ Poland
}

Submitted: 12 July 2019

Accepted: 15 April 2020

Arch Med Sci

DOI: https://doi.org/10.5114/aoms.2020.95424

Copyright (c) 2020 Termedia \& Banach

\section{Abstract}

Introduction: Chronic kidney disease and end-stage renal disease have been found to be caused by diabetes. More recently, the renal tubulointerstitium has been increasingly assumed to play a role in the pathogenesis of diabetic nephropathy with prolonged exposure to a variety of metabolic and haemodynamic injuring factors associated with sustained hyperglycaemia as contributing factors. This study aimed to investigate whether maternal diabetes could be the factor affecting kidney function in a newborn with the use of neutrophil gelatinase-associated lipocalin (NGAL) and kidney injury molecule-1 (KIM-1) - biomarkers of renal injury.

Material and methods: The study included 138 full-term newborns: 50 newborns from diabetic pregnancies and 88 healthy newborns. The concentrations of NGAL and KIM-1 were determined in urine in the first or the second day of life with a commercially available ELISA kit.

Results: Considerably higher urine level of NGAL (25.7 (11.8-40.8)) and NGAL/cr. (29.1 (19.1-47.4)) in babies from diabetic pregnancies has been found when compared to the reference group (16.74 (9.9-27.5)) and (21.9 $(14.6-29.8))(p=0.01, p<0.01)$ respectively. We also found a significantly higher urine level of NGAL (27.8 (13.6-44.2)), NGAL/cr. (31.9 (17.6-57.4)), and $\mathrm{KIM}-1 / \mathrm{cr}$. (2.6 (1.6-5.5)) in babies of diabetic mothers treated with insulin when compared to the reference group (16.7 (9.9-27.5)), (21.9 (14.6$29.8)),(1.9(0.8-3.2)),(p=0.01, p=0.02, p=0.02)$, respectively.

Conclusions: Based on the results of this study, we indicate for the first time that maternal diabetes mellitus during pregnancy may be considered as the cause of tubular kidney damage in newborns.

Key words: diabetes mellitus, kidney injury, biomarkers.

\section{Introduction}

Diabetes mellitus, a chronic metabolic disease, is believed to be the most common hormonal disease in the world [1]. It is estimated that diabetes can cause chronic kidney disease and end-stage renal disease [2]. It has also been increasingly assumed that the renal tubulointerstitium takes part in the pathogenesis of diabetic nephropathy with extended exposure to a variety of metabolic and haemodynamic injuring

\author{
Corresponding author: \\ Monika Kamianowska \\ MD, PhD \\ Department \\ of Neonatology and \\ Neonatal Intensive Care \\ Medical University \\ 24 A M. Skłodowskiej-Curi St \\ 15-276 Białystok, Poland \\ Phone: +48 857468498 \\ E-mail: monikakamm@wp.pl
}


factors associated with sustained hyperglycaemia as a contributing factor [3].

Diabetes mellitus (type 1 diabetes, type 2 diabetes, gestational diabetes) is a frequent medical complication in pregnancy [4]. Not only is the mother adversely affected but also the child, causing early perinatal complications and long-term consequences. There is vast body of evidence that, regardless of diabetes and hyperglycaemia during pregnancy, the risk of adverse events increases $[5,6]$.

Neutrophil gelatinase-associated lipocalin (NGAL) produced in epithelial cells and neutrophils in most tissues is a small (25-kD) protein. It is a marker of renal tubular damage [7]. Also, kidney injury molecule-1 (KIM-1), a transmembrane protein present only in the proximal tubules of injured human kidneys, can be considered as a marker of acute proximal tubular kidney damage [8].

Until now, no study concerning the impact of diabetes during pregnancy on the functions of newborns' kidneys has been conducted using early biomarkers of kidney damage.

The purpose of the study was to test the differences in concentrations of urine NGAL and KIM-1 between newborns exposed to maternal diabetes during pregnancy and newborns from normal pregnancies.

\section{Material and methods}

\section{Patient recruitment and sample collection}

In the prospective study 138 neonates were included. They were born on time at the Department of Perinatology and hospitalised at the Department of Neonatology and Intensive Care, Medical University of Bialystok, Poland at a rooming-in ward. Fifty newborns were from pregnancies complicated by diabetes mellitus (11 by type 1 diabetes, 17 by gestational diabetes requiring insulin therapy, 22 by gestational diabetes requiring a diet therapy, who were diagnosed using WHO 2013 criteria [9]).

Eighty-eight healthy newborns resulted from normal pregnancies with no prenatal and perinatal complications. Newborns included in the study met the following criteria: normal physical examination and normal prenatal ultrasound examination of the kidney. Neonates with a history of any congenital anomaly, Apgar score lower than 8 in the first minute of birth, respiratory disorders, heart illnesses, intrauterine infections, and higher markers of inflammation were not included in the analysis.

The Local Bioethics Committee of the Medical University of Bialystok approved the protocol of the study. We conducted the procedures according to the Helsinki Declaration. We received written informed consent from parents of all the newborns before the study.

Samples of urine using single-use sterile bags (UNIDEM, London) were taken from the study group. We collected urine and blood samples on the first or second day of life. The blood samples were drawn during routine practice in the unit, using S-Monovette $1.2 \mathrm{ml}$, Clotting Activator/Serum test tubes (Sarstedt AG \& Co, Germany). The samples of serum and urine after centrifugation were kept in the fridge $\left(4^{\circ} \mathrm{C}\right)$ for a maximum of $2 \mathrm{~h}$ and then frozen at $-80^{\circ} \mathrm{C}$. Repeated freeze-thaw cycles were avoided.

\section{Determination of urine NGAL}

We measured the levels of urine NGAL using an ELISA kit (R\&D Systems, Minneapolis, MN, USA \& Canada), which is commercially available. We measured the levels according to the manufacturer's instructions, with results in nanograms per millilitre. The detection limit was $<0.1 \mathrm{ng} / \mathrm{ml}$. The mean intra- and inter-assay coefficients of variation (CV) for urine NGAL were 3.4 and $11.4 \%$, respectively.

\section{Determination of urine $\mathrm{KIM}-1$}

We determined the urine concentrations of KIM-1 using an ELISA kit (R\&D Systems, Minneapolis, MN, USA \& Canada), which is commercially available. We measured the concentrations according to the manufacturer's instructions, with results in nanograms per millilitre $(\mathrm{ng} / \mathrm{ml})$. The limit of detection of KIM-1 levels was $0.07 \mathrm{ng} / \mathrm{ml}$.

We normalised the urine concentrations of NGAL and KIM-1 for urine concentration of creatinine measured by Jaffé's method (UNGAL-1/cr., uKIM-1/cr.) to account for a potential confounding effect of urine dilution. It was expressed in nanograms per milligram of creatinine ( $\mathrm{ng} / \mathrm{mg} \mathrm{cr}$.). The level of serum creatinine was determined by Jaffe's method and shown in milligrams per decilitre. We calculated the estimated glomerular filtration rate (GFR) according to Schwartz formula (eGFR $=0.45$ $\times L / S c r$., where $L$ is length in centimetres, and Scr. is serum creatinine in milligrams per decilitre).

\section{Statistical analysis}

We analysed the results using Statistica 12.0 package (StatSoft, USA). We showed discrete variables as counts (percentage), whereas continuous variables as median and quartiles (Q1-Q3). To determine the normal distribution, we used Shapiro-Wilk test, and if the data were not normally distributed, we used Mann-Whitney $U$-test for intergroup comparisons of continuous variables.

We compared the distributions of discrete variables with the $\chi^{2}$ test. Spearman's coefficients of 
rank correlation measured the direction and the power of association between the concentrations of KIM-1 and other variables. We analysed the results of all the tests significant at $p<0.05$.

\section{Results}

In total, 138 full-term newborns were included in the study. Fifty neonates (33 boys, 17 girls) were exposed to maternal diabetes during pregnancy (D). Eighty-eight healthy newborns (44 boys, 44 girls) -the reference group (R) - were born from normal pregnancies with no prenatal and perinatal complications. Both groups were sex-matched ( $p>0.05)$. We also divided the examined group (D) into two subgroups: babies born from diabetic mothers treated only with a diet (D1) and with insulin therapy (D2). There were no complications in the pregnancies of mothers with diabetes. Forty mothers had glycated haemoglobin $\left(\mathrm{HbA}_{1 \mathrm{c}}\right)$ $<6.0 \%$ without hypoglycaemia. The rest of them (mothers with gestational diabetes treated with a diet) reported that they had regularly measured blood glucose concentrations during the day and they were normal (fasting blood glucose levels < $5.3 \mathrm{mmol} / \mathrm{l}$ (95 mg/dl), $2 \mathrm{~h}$ after a meal $<6.7 \mathrm{mmol} / \mathrm{l}(120 \mathrm{mg} / \mathrm{dl}))$.

The serum glucose concentrations in all newborns were normal.

In Table I we present the patients' demographics, median values, and interquartile range (IQR) of measured parameters.

Newborns from normal and those from diabetic pregnancies were born on time; however, the gestational age of neonates in group $D$ was lower $(p<0.01)$. There were no significant differences between the parameters of physical development (birth weight, length, head and chest circumference). Significantly higher concentrations of NGAL and NGAL/cr. in urine were found in newborns from diabetic pregnancies comparing to the reference group (R) ( $p<0.02 ; p<0.01$, respectively). Also, urine KIM-1 was higher in group $D$ compared to the reference group $(R)$, but the differences did not differ considerably. No significant differences in the concentration of creatinine and the estimated GFR in serum were found.

In Table II we compared measured parameters in subgroups on a diet (D1) and on insulin therapy (D2) with the reference group (R). Comparing the parameters between group D1 and R we only found that the gestational age in group D1 was lower than in group $\mathrm{R}(p<0.05)$. No statistically relevant differences were observed in the concentrations of NGAL and KIM-1 between groups D1 and R. However, when comparing the subgroup on insulin therapy (D2) with group R, more statistical discrepancies were found between the groups than when the whole diabetic group (D) was compared with the reference group (R). In subgroup D2 the gestational age was significantly lower $(p<0.01)$. There was considerably higher concentration of NGAL, and also NGAL/cr. in the urine was significantly higher, in group D2 compared to group $\mathrm{R}(p<0.02$ and $p<0.03$, respectively) (Figure 1 ).

Statistically relevant differences were also found in the $\mathrm{KIM} / \mathrm{cr}$. ratio between these two groups. The median concentrations of $\mathrm{KIM}-1$ were

Table I. Characteristics of the study population - newborns from diabetic (D) and normal pregnancies (R)

\begin{tabular}{|c|c|c|c|}
\hline \multirow[t]{2}{*}{ Parameter } & Diabetes (D) $(n=50)$ & Reference group (R) $(n=88)$ & \multirow[t]{2}{*}{$P$-value } \\
\hline & \multicolumn{2}{|c|}{ Median (Q1-Q3) } & \\
\hline Gestational age [weeks] & $39(38-39)$ & $39(39-40)$ & 0.0002 \\
\hline Gender (boys/girls) & $33 / 17$ & $44 / 44$ & 0.069 \\
\hline Birth weight [g] & $3400(3000-3860)$ & $3450(3165-3800)$ & NS \\
\hline Length $[\mathrm{cm}]$ & $56(54-58)$ & $56(54-57)$ & NS \\
\hline Head circuit [cm] & $34(33-35)$ & $34(33-35)$ & NS \\
\hline Chest circuit [cm] & $33(32-35)$ & $34(33-35)$ & NS \\
\hline uNGAL [ng/ml] & $25.74(11.84-40.81)$ & $16.74(9.99-27.47)$ & 0.015 \\
\hline uNGAL/creatinine [ng/mg cr.] & $29.12(19.12-47.41)$ & $21.91(14.57-29.77)$ & 0.007 \\
\hline uKIM-1 [ng/ml] & $1.43(0.80-4.64)$ & $1.29(0.63-2.52)$ & NS \\
\hline uKIM-1/creatinine [ng/mg cr.] & $2.23(1.09-4.09)$ & $1.87(0.84-3.26)$ & NS \\
\hline Serum creatinine [mg/dl] & $0.67(0.56-0.83)$ & $0.61(0.54-0.70)$ & NS \\
\hline $\mathrm{eGFR}\left[\mathrm{ml} / \mathrm{min} / 1.73 \mathrm{~m}^{2}\right]$ & $37.85(29.30-43.60)$ & $40.15(35.00-46.70)$ & NS \\
\hline
\end{tabular}

UNGAL - urine neutrophil gelatinase-associated lipocalin, UKIM-1 - urine kidney injury molecule-1, cr. - creatinine, eGFR - estimated glomerular filtration rate, $p$-diabetes - reference group. 
Table II. Comparison of examined parameters in subgroups on diet (D1) and on insulin therapy (D2) with the reference group (R)

\begin{tabular}{|lccccc|}
\hline Parameter & $\begin{array}{c}\text { Diabetes (D1) } \\
(n=22)\end{array}$ & $\begin{array}{c}\text { Diabetes (D2) } \\
(n=28)\end{array}$ & $\begin{array}{c}\text { Reference(R) } \\
(n=44)\end{array}$ & P-value ${ }_{1}$ & $P_{\text {-value }}$ \\
\cline { 2 - 4 } & \multicolumn{3}{c}{ Median (Q1-Q3) } & & \\
\hline Gestational age [weeks] & $39(38-40)$ & $38(37.5-39)$ & $39(39-40)$ & 0.045 & 0.0002 \\
\hline Gender (boys/girls) & $14 / 8$ & $19 / 9$ & $44 / 44$ & 0.251 & 0.098 \\
\hline Birth weight [g] & $3300(3020-3750)$ & $3520(3000-3865)$ & $3450(3165-3800)$ & NS & NS \\
\hline Length [cm] & $56(54-58)$ & $56(54-57.5)$ & $56(54-57)$ & NS & NS \\
\hline Head circuit [cm] & $34(32-35)$ & $33.5(33-35)$ & $34(33-35)$ & NS & NS \\
\hline Chest circuit [cm] & $33.5(32-35)$ & $33(32-35)$ & $34(33-35)$ & NS & NS \\
\hline uNGAL [ng/ml] & $25.15(11.61-39.54)$ & $27.78(13.58-44.27)$ & $16.74(9.99-27.47)$ & NS & 0.010 \\
\hline uNGAL/creatinine [ng/mg cr.] & $26.30(20.22-44.34)$ & $31.88(17.63-57.42)$ & $21.91(14.57-29.77)$ & NS & 0.022 \\
\hline uKIM-1 [ng/ml] & $1.08(0.57-2.08)$ & $1.758(0.88-5.66)$ & $1.289(0.63-2.52)$ & NS & NS \\
\hline uKIM-1/creatinine [ng/mg cr.] & $1.41(1.03-2.99)$ & $2.64(1.58-5.56)$ & $1.87(0.84-3.26)$ & NS & 0.019 \\
\hline Serum creatinine [mg/dl] & $0.64(0.51-0.71)$ & $0.70(0.60-0.90)$ & $0.61(0.54-0.70)$ & NS & NS \\
\hline eGFR [ml/min/1.73 m²] & $41.30(36.80-49.70)$ & $36.30(27.75-41.90)$ & $40.15(35.00-46.70)$ & NS & NS \\
\hline
\end{tabular}

UNGAL - urine neutrophil gelatinase-associated lipocalin, UKIM-1 - urine kidney injury molecule-1, cr. - creatinine, eGFR - estimated glomerular filtration rate, $p_{1}$-diabetes (D1) - reference group, $p_{2}$-diabetes (D2) - reference group.

higher in group D2 compared to the reference group $R$, but these differences were not statistically significant.

Because the groups were sex-matched, the association between female gender and higher concentrations of NGAL and also NGAL/cr. in the urine was found both in the whole group of neonates taking part in the study $(p<0.001)$ and in the group of neonates from diabetic pregnancies $(p<0.01)$ (Figure 2). The concentration of KIM-1 did not depend on sex.

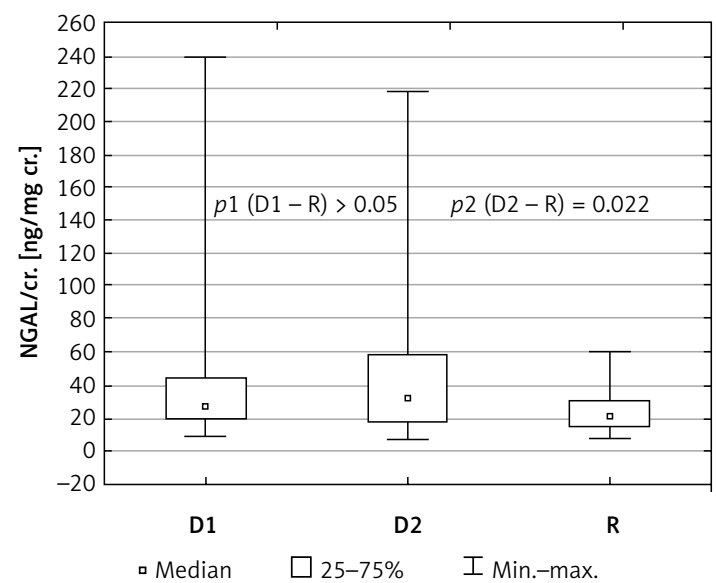

Figure 1. Urine NGAL/cr. depending on type of diabetes treatment

UNGAL - urine neutrophil gelatinase-associated lipocalin, cr. - creatinine, D1 - subgroup on diet therapy, D2 subgroup on insulin therapy, $R$ - reference group.
There were negative correlations between the urine NGAL/cr. and the week of pregnancy $(R=$ -0.196, $p=0.02)$, birth weight $(R=-0.381, p<$ $0.001)$, head circumference $(R=-0.333, p<0.001)$, and chest circumference $(R=-0.330, p<0.001)$. These correlations were not found in $\mathrm{KIM}-1 / \mathrm{cr}$.

Newborns from normal and from diabetic pregnancies were born on time; however, the gestational age of newborns from group $D$ was lower $(p<0.01)$. Newborns from pregnancies complicated by diabetes were born in our hospital a little

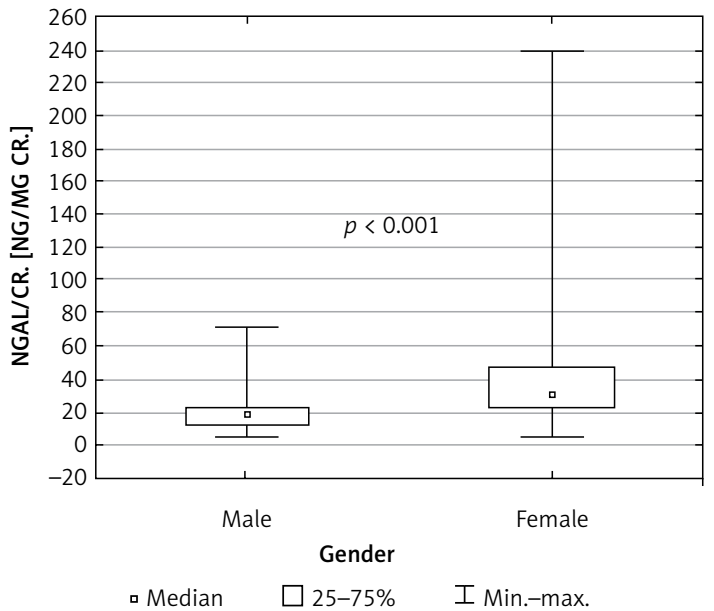

Figure 2. Urine NGAL/cr. depending on gender in the whole study population - newborns from diabetic (D) and normal pregnancies (R)

UNGAL - urine neutrophil gelatinase-associated lipocalin, cr. - creatinine. 
earlier than normal pregnancies. After observation, we came to the conclusion that only urine $\mathrm{NGAL/cr}$. correlated negatively with the week of pregnancy.

We used the factors that were found to have had a significant correlation with the urine NGAL/ cr. ratio as explanatory variables to create multiple regression models. Some correlated variables were removed to reduce the impact of multicollinearity. In this model the remaining three parameters (gestational age, birth weight, male gender) comprised over $22 \%$ of the variations in the NGAL/cr. ratio level $\left(R=0.4754, R^{2}=0.2261, p<0.01\right)$.

\section{Discussion}

Nowadays, diabetes is more often regarded as the main cause of chronic renal failure, with many patients progressing to end-stage renal illness needing dialysis or transplantation [10]. The damage affects not only the glomerulus but also the renal tubules [11]. Histological studies show that proximal tubular basement membrane width is already thickened in normoalbuminuric diabetic patients compared to healthy controls [12]. Fioretto et al. observed that in microalbuminuric diabetic patients with similar GFR only $29 \%$ of patients had typical histological glomerular features of diabetic nephropathy, whereas $42 \%$ had severe tubulointerstitial lesions disproportional to mild glomerular involvement [13].

Not only are the early perinatal, diabetescaused complications further complications of pregnancy, but they also have long-term consequences. The study by Nelson et al. provided evidence that maternal hyperglycaemia may lead to adult disease in offspring [14]. They concluded that the risk of elevated urine albumin excretion in diabetic Pima Indians is increased by exposure to a diabetic intrauterine environment [14]. Yan et al. observed early renal dysfunction of male newborns born by mothers with diabetes and moderate hyperglycaemia [15]. Also, in the urine of newborns born from diabetic mothers the presence of $\mathrm{N}$-acetyl-b-D-glucosaminidase, an enzyme which reflects the degree of proximal tuber necrosis, was affected [15].

Patients with diabetes have elevated concentrations of urine NGAL and KIM-1, even if albuminuria is not observed [16-18]. The values of urine NGAL and KIM-1 in newborns born from pregnancies complicated by diabetes mellitus have been determined for the first time in this prospective study. Babies born by mothers with diabetes are exposed to permanent or periodic (in the case of balanced diabetes) hyperglycaemia. In the study on foetuses with exposure to maternal diabetes during pregnancy, Taricco et al. made a similar observation. These babies had higher concentrations of umbilical glucose, even if maternal glucose levels were normal, as well as the reduction in oxygen saturation and oxygen content with higher lactate concentration. It showed altered foetal metabolism [19]. Hyperglycaemia inducing the production of reactive oxygen species, leading to the accumulation of advanced glycosylated end-products and activation of protein kinase C, can in turn lead to tissue injury not only to the glomerulus, in particular podocytes, but also to the tubulointerstitium [20-22]. There are structural changes in the proximal tubules, such as tubular atrophy, interstitial fibrosis, and peritubular capillary rarefaction [19].

Unfortunately, there are no data on the levels of NGAL and $\mathrm{KIM}-1$ in babies from pregnancies complicated by diabetes in the literature. Higher excretion of urine NGAL was, however, found in early-stage diabetes, e.g. type 1 in children [17, 23]. Taking into consideration the fact that urine $\mathrm{N}$-acetyl-b-D-glucosaminidase is a marker of renal tubular damage, the observation made by Yan et al., who found an increase in excretion of this enzyme in the offspring of diabetic rats, may prove the importance of looking for markers of the coils' damage in the group of newborns of mothers with diabetes [15]. In the study, we observed that the median urine NGAL and urine NGAL/cr. levels were significantly higher in newborns born by mothers with diabetes $(p<0.02 ; p<0.01$ ), and that the difference of urine NGAL concentration was even more significant between group D2 and the reference group $(p=0.01)$ and similar for NGAL/cr. $(p<0.02)$. We observed higher excretion of urine only in the group of babies from pregnancies complicated by diabetes treated with insulin compared to the reference group. It was probably associated with a higher risk of hyperglycaemia in type 1 diabetes compared to gestational diabetes. Analysing the results, we assumed at NGAL is a more sensitive and early marker of tubular damage than KIM-1. Other authors also found such results. The studies in newborns with AKI confirmed that NGAL was an important early biomarker of AKI, but data on KIM-1 were not conclusive [24-29]. Some authors indicated its statistically significant role as an early AKI biomarker [25], while others did not $[26,27]$. In some publications the level of NGAL elevated rapidly after the activation of a damaging factor, whereas the increase in KIM-1 concentration was associated with the occurrence of proteinuria $[28,29]$.

It should also be mentioned that the level of NGAL depends on gender and is much lower in boys, which was proved in studies involving both children and adults [30, 31]. During our study, we found that urine NGAL concentration depended on sex and was higher in female gender both in the $D$ and $R$ groups. These differences were not 
found in KIM-1 concentrations. Multifactorial regression also confirmed our observation, in which we showed that gender had the biggest impact on the value of NGAL/cr.

It is also worth emphasising the fact that babies qualified for the study were born from pregnancies complicated by diabetes but properly compensated. Newborns were born on time, and no features of diabetic fetopathy were observed. Therefore, it should be assumed that changes in the kidney tubules might not be very advanced. We should note that in children from group $D$, we did not observe hyperfiltration, and there were no differences in the eGFR between them and healthy controls. We found a negative correlation between NGAL and KIM-1, but it was not statistically significant. It was probably because diabetic changes in the kidneys were not advanced.

We are planning a further multicentre study, in which we will analyse markers of tubular damage in babies born with large birth weight because of hyperglycaemia caused by diabetes in pregnancy.

In conclusion, urine $\mathrm{NGAL/cr}$. is statistically significantly higher in newborns exposed to maternal diabetes during pregnancy compared to babies from normal pregnancies. It may suggest subclinical tubular dysfunction in these babies. Urine NGAL is a more sensitive marker than KIM-1 in these newborns. The level of urine NGAL is higher in female babies than in male newborns. In children with normal birth weight born from diabetic pregnancies, eGFR is normal and we do not observe hyperfiltration.

\section{Acknowledgments}

The grant of the Medical University of Bialystok funded this study.

\section{Conflict of interest}

The authors declare no conflict of interest.

\section{References}

1. Kayar Y, Agin M. The relationship between demographic and anthropometric characteristics and diabetic complications and number of hospitalizations in hospitalized diabetic patients. Arch Med Sci Civil Dis 2019; 4: e7-15.

2. Lim AKH. Diabetic nephropathy - complications and treatment. Int J Nephrol Renovasc Dis 2014; 15: 361-81.

3. Thomas MC, Burns WC, Cooper ME. Tubular changes in early diabetic nephropathy. Adv Chronic Kidney Dis 2005; 12: 177-86

4. Simeoni U, Barker DJ. Offspring of diabetic pregnancy: long-term outcomes. Semin Fetal Neonatal Med 2009; 14: 119-24.

5. Balsells M, García-Patterson A, Gich I, Corcoy R. Maternal and fetal outcome in women with type 2 versus type 1 diabetes mellitus: a systematic review and metaanalysis. J Clin Endocrinol Metab 2009; 94: 4284-91.
6. Cyganek K, Skupien J, Katra B, et al. Risk of macrosomia remains glucose-dependent in a cohort of women with pregestational type 1 diabetes and good glycemic control. Endocrine 2017; 55: 447-55.

7. Mitsnefes MM, Kathman TS, Mishra J, et al. Serum neutrophil gelatinase-associated lipocalin as a marker of renal function in children with chronic kidney disease. Pediatr Nephrol 2007; 22: 101-8.

8. Nielsen SE, Schjoedt KJ, Astrup AS, et al. Neutrophil gelatinase-associated lipocalin (NGAL) and kidney injury molecule 1 (KIM1) in patients with diabetic nephropathy: a cross-sectional study and the effects of lisinopril. Diabet Med 2010; 27: 1144-50.

9. World Health Organization. Diagnostic Criteria and Classification of Hyperglycemia First Detected in Pregnancy. Geneva, World Health Org. 2013. WHO/NMH/ MND/13.

10. Molitch ME, DeFronzo RA, Franz MJ, et al.; American Diabetes Association. Nephropathy in diabetes. Diabetes Care 2004; 1: S79-83.

11. Vallon $\mathrm{V}$. The proximal tubule in the pathophysiology of the diabetic kidney. Am J Physiol Regul Integr Comp Physiol 2011; 300: R1009-22.

12. Brito PL, Fioretto P, Drummond K, et al. Proximal tubular basement membrane width in insulin-dependent diabetes mellitus. Kidney Int 1998; 53: 754-61.

13. Fioretto P, Mauer M, Brocco E, et al. Patterns of renal injury in NIDDM patients with microalbuminuria. Diabetologia 1996; 39: 1569-76.

14. Nelson RG, Morgenstern H, Bennett PH. Intrauterine diabetes exposure and the risk of renal disease in diabetic Pima Indians. Diabetes 1998; 47: 1489-93.

15. Yan J, Li X, Su R, Zhang K, Yang H. Long-term effects of maternal diabetes on blood pressure and renal function in rat male offspring. PLoS One 2014; 9: e88269.

16. Lacquaniti A, Donato V, Pintaudi B, et al. "Normoalbuminuric" diabetic nephropathy: tubular damage and NGAL. Acta Diabetol 2013; 50: 935-42.

17. YürükYıldırım Z, Nayır A, Yılmaz A, Gedikbaşı A, Bundak R. Neutrophil gelatinase-associated lipocalin as an early sign of diabetic kidney injury in children. J Clin Res Pediatr Endocrinol 2015; 7: 274-79.

18. Nauta FL, Boertien WE, Bakker SJ, et al. Glomerular and tubular damage markers are elevated in patients with diabetes. Diabetes Care 2011; 34: 975-81.

19. Taricco E, Radaelli T, Rossi G, et al. Effects of gestational diabetes on fetal oxygen and glucose levels in vivo. BJOG 2009; 116: 1729-35.

20. Raptis AE, Viberti G. Pathogenesis of diabetic nephropathy. Exp Clin Endocrinol Diabetes 2001; 109: S424-37.

21. Cao Z, Cooper ME. Pathogenesis of diabetic nephropathy. J Diabetes Investig 2011; 2: 243-7.

22. Gilbert RE. Proximal tubulopathy: prime mover and key therapeutic target in diabetic kidney disease. Diabetes 2017; 66: 791-800.

23. Abd El Dayem S, El BohyAel M, El Shehaby A. Value of the intrarenal arterial resistivity indices and different renal biomarkers for early identification of diabetic nephropathy in type 1 diabetic patients. J Pediatr Endocrinol Metab 2016; 29: 273-9.

24. Abdelhady S, Gawad ERA, Haie OMA, Mansour Al. Usefulness of serum and urinary neutrophil gelatinaseassociated lipocalin in detecting acute kidney injury in asphyxiated neonates. Int J Med Health Sci 2016; 1: 4230-6.

25. Cao XY, Zhang HR, Zhang W, Chen B. Diagnostic values of urinary netrin-1 and kidney injury molecule-1 
for acute kidney injury induced by neonatal asphyxia.

Zhongguo Dang Dai Er Ke Za Zhi 2016; 18: 24-8.

26. Stojanović VD, Barišić NA, Vučković NM Doronjski AD,

PecoAntić AE. Urinary kidney injury molecule-1 rapid test predicts acute kidney injury in extremely low-birthweight neonates. Pediatr Res 2015; 78: 430-5.

27. Askenazi DJ, Koralkar R, Patil N, Halloran B, Ambalavanan N, Griffin R. Acute kidney injury urine biomarkers in very low-birth-weight infants. Clin J Am Soc Nephrol 2016; 11: 1527-35.

28. Panduru NM, Sandholm N, Forsblom C, et al. Kidney injury molecule- 1 and the loss of kidney function in diabetic nephropathy: a likely causal link in patients with type 1 diabetes. Diabetes Care 2015; 38:1130-7.

29. Vanmassenhove J, Vanholder R, Nagler E, Van Biesen W. Urinary and serum biomarkers for the diagnosis of acute kidney injury: an in-depth review of the literature. Nephrol Dial Transplant 2013; 28: 254-73.

30. Askenazi DJ, Koralkar R, Levitan EB, et al. Baseline values of candidate urine acute kidney injury biomarkers vary by gestational age in premature infants. Pediatr Res 2011; 70: 302-6.

31. Pennemans V, Rigo JM, Faes C, Reynders C, Penders J, Swennen Q. Establishment of reference values for novel urinary biomarkers for renal damage in the healthy population: are age and gender an issue? Clin Chem Lab Med 2013; 51: 1795-802. 